\title{
Zebrafish enhancer trap line recapitulates embryonic aquaporin 1a expression pattern in vascular endothelial cells
}

\author{
KIRA REHN ${ }^{1}$, KUAN SHEN WONG ${ }^{1}$, DARIUS BALCIUNAS ${ }^{2}$ and SAULIUS SUMANAS ${ }^{*}, 1,3$ \\ ${ }^{1}$ Division of Developmental Biology, Cincinnati Children's Hospital Medical Center, Cincinnati, OH, ${ }^{2}$ Department of \\ Biology, Temple University, Philadelphia, PA and ${ }^{3}$ Division of Hematology / Oncology, Cincinnati Children's Hospital \\ Medical Center, University of Cincinnati Medical School, Cincinnati, OH, USA
}

\begin{abstract}
Aquaporin 1 (Aqp1) is a water channel protein, expressed widely in microvascular endothelial cells and implicated in mammalian tumor angiogenesis. However, its developmental expression has not yet been characterized in great detail. An enhancer trap screen was performed using a Tol2-derived GFP reporter in zebrafish embryos. An insertional Et(GBT-B1)tpl1 line was identified that has reporter insertion in the vicinity of the aqp1a gene. We further characterized the embryonic expression pattern of this GFP reporter line, as well as that of endogenous aqp1a. Both endogenous aqp1a and reporter GFP expression were restricted to the vascular endothelial cells within the dorsal aorta, cranial, intersegmental and other secondary vessels, but were absent in the axial venous vasculature. In addition, endogenous aqp1a expression was observed in both primitive and definitive hematopoietic erythroid progenitors, as well as in the otic vesicle, swim bladder, pneumatic duct, intestine and a subset of neurons within the retina and the midbrainhindbrain region. We further show that gata1 and etsrp/etv2 function is required for hematopoietic and endothelial aqp1a expression, respectively. Aqp1a expression is restricted to endothelial and erythroid cells during early embryogenesis. The transgenic Et(GBT-B1)tpl1 line recapitulates endogenous endothelial aqp1a expression. Because currently very few reporter lines can differentiate between arterial and venous endothelial cells, the Et(GBT-B1)tpl1 transgenic line and characterization of the aqp1a expression pattern will be useful for future studies of endothelial and arterial-venous differentiation.
\end{abstract}

KEY WORDS: aquaporin, angiogenesis, enhancer trap, vascular endothelial, erythroid, tumor

Aquaporin 1 is a water channel protein expressed in vascular endothelial cells in multiple different vertebrates. Its strong expression has been observed in microvascular endothelium in rats and mice within adult kidney, lung, skin, secretory glands, pleura and peritoneum (Hasegawa et al., 1994, Nielsen et al., 1993a, Verkman, 2006). In addition, AQP1 is a major component of red blood cell and renal tubule membranes (Harris et al., 1991, Nielsen et al., 1993b). AQP1 is expressed strongly in proliferating microvessels in multiple different tumors in humans including mammary carcinomas and glioblastomas, brain tumors such as astrocytomas and metastatic carcinomas as well as bone marrow microvessels in patients with active multiple myeloma (Endo et al., 1999, Saadoun et al., 2002, Vacca et al., 2001, Verkman et al., 2008). Endothelial AQP1 expression has been also observed within the chick cho- rioallantoic membrane (CAM) (Ribatti et al., 2002). Disruption of AQP1 function results in impaired tumor microvessel proliferation in mice tumor model, resulting in extensive tumor necrosis (Saadoun et al., 2005). AQP1 function is required for endothelial cell motility, as demonstrated by mammalian cell culture and CAM

\footnotetext{
Abbreviations used in this paper: Aa, aortic arches; Aqp, aquaporin; CAM, chorioallantoic membrane; CrDI, internal carotid artery; DA, dorsal aorta; DLAV, dorsal longitudinal anastomotic vessel; Dpf, days-post-fertilization; GFP, green fluorescent protein; Hpf, hours-post-fertilization; ICM, intermediate cell mass; ISV, intersegmental Vessels; LDA, lateral dorsal aorta; $\mathrm{MCeV}$, middle cerebral vein; MO, morpholino; PCV, posterior cardinal vein; $\mathrm{PHBC}$, primordial hindbrain channel; PICA, primitive internal carotid artery; PMBC, primordial midbrain channel; RT-PCR, reverse transcriptase polymerase chain reaction.
}

*Address correspondence to: Saulius Sumanas. Division of Developmental Biology, CCHMC, 3333 Burnet Ave., Cincinnati, OH 45229 , USA.

Fax: +1-513-636-4317. E-mail: saulius.sumanas@ cchmc.org - web: http://www.cincinnatichildrens.org/research/div/dev-biology/fs/fac/saulius-sumanas.htm

Supplementary Material (two figures) for this paper is available at: http://dx.doi.org/10.1387/ijdb.103249kp

Accepted: 11 January 2011. Final, author-corrected PDF published online: 6 September 2011.

ISSN: Online 1696-3547, Print 0214-6282

(C) 2011 UBC Press

Printed in Spain 
assays (Camerino et al., 2006, Saadoun et al., 2005). While these results demonstrate a critical role for AQP1 function during tumor angiogenesis, AQP1-null mice are viable (Saadoun et al., 2005), therefore its role in embryonic development and angiogenesis, if any, is currently unknown.

During fetal and prenatal rat development, AQP1 expression has been observed in choroid plexus, periostium, endocardium and cornea (Bondy et al., 1993). During the early development of killifish Fundulus heteroclitus aqp1a is expressed in the developing blood vessels including the caudal artery and intersegmental vessels (Tingaud-Sequeira et al., 2009). However, it is not known if the embryonic vascular expression pattern of aqp1 is evolutionarily conserved and comprehensive analysis of embryonic aqp 1 expression during different developmental stages is still not available.

Zebrafish has emerged as an advantageous model system to study embryonic vascular development. Similar to other vertebrates, zebrafish contains homologs for all thirteen different mammalian Aqp family members, which in some cases have undergone genomic duplication (Tingaud-Sequeira et al., 2010). In zebrafish, similar to other teleost fish, two aqp1 homologs are present in close genomic location. Aqp1b / aqp1a.2 gene is located immediately downstream of aqp 1a / aqp 1a.1 within the chromosome 2 (Tingaud-Sequeira et al., 2008). DNA and protein sequences of the two zebrafish aqp1 homologs have been previously described (Tingaud-Sequeira et al., 2008). Interestingly, based on RT-PCR analysis, aqp1a is expressed in multiple zebrafish adult tissues while adult aqp $1 b$ expression is restricted to the ovary, testis and brain (Tingaud-Sequeira et al., 2010, Tingaud-Sequeira et al., 2008). However, embryonic expression of either zebrafish aqp1 homolog has not been previously investigated.

Arterial-venous $(A-V)$ differentiation is a critical step in the establishment of functional vasculature. In zebrafish, similar to other vertebrates, $A-V$ differentiation is initiated prior to the establishment of circulation during formation of the major axial vessels, the dorsal aorta and the posterior cardinal vein (Jin et al., 2005, Zhong et al., 2001, Zhong et al., 2000). According to the current model, notochord-derived Sonic Hedgehog induces expression of the vascular endothelial growth factor (Vegf) within the medial part of the somites. Vegf through PLC $\gamma$-dependent ERK phosphorylation activates the expression of Notch and its ligand Delta within the arterial precursors which then act to promote arterial and repress venous marker expression (Hong et al., 2006, Lawson et al., 2001, Lawson et al., 2002). While multiple arterial and venous specific markers have been previously identified in zebrafish including an aquaporin homolog aqp8 which is expressed in the arterial but not venous endothelial progenitors (Sumanas etal., 2005), it is currently not known if aqp1 exhibits differential A-V expression pattern.

Enhancer trap screens have been previously used to generate new fluorescent reporter lines and to identify novel gene expression patterns (Asakawa et al., 2008, Balciunas et al., 2004, Ellingsen et al., 2005, Parinov et al., 2004, Poon et al., 2010). While there are multiple vascular endothelial specific lines generated either by traditional transgenesis or enhancer trap methods, most of them show ubiquitous endothelial expression and are not useful to specifically identify arterial or venous blood vessels.

In this study, we report generation of a new GFP reporter line Et(GBT-B1)tpl1 discovered in a Tol2-mediated enhancer trap screen. This line displays embryonic GFP expression restricted to the arterial axial vessels as well as cranial and intersegmental vessels while excluded from the axial venous vessels. We further show that the reporter insertion is located in the vicinity of aqp1ain the zebrafish genome. We characterize developmental expression of aqp1awhich is primarily restricted to the arterial vasculature including the dorsal aorta and is excluded from the cardinal vein, similar to the reporter GFP expression pattern. In addition, endogenous aqp1a is also expressed in erythroid progenitors. These findings suggest that aqp1a may play a specific function during embryonic endothelial and / or hematopoietic development. Furthermore, Et(GBT-B1) tpl1 line will be a useful tool to study endothelial development and arterial-venous differentiation.

\section{Results}

Tol2 mediated transgenesis was used in an enhancer trap screen to generate reporter lines that display novel expression patterns. Transgenesis was performed using GBT-B1 vector which is designed for both enhancer trap and gene trap. To trap novel enhancers, GBT-B1 contains zebrafish $\beta$-actin minimal promoter in front of eGFP (see Experimental Procedures and Suppl. Fig. S1). Detailed construction of the vector and the screening procedure will be presented elsewhere (Balciuniene et al., in preparation).

One of the generated lines, Et(GBT-B1)tpl1, displayed specific GFP fluorescence within vascular endothelial cells starting at the 26 hours-post-fertilization (hpf) stage (data not shown). At 31-48 hpf stages, GFP expression was observed within the dorsal aorta, intersegmental vessels, dorsal longitudinal anastomotic vessel, the plexus region of the cardinal vein and cranial vasculature (Fig. 1A-E). Interestingly, axial vessel expression was restricted to the dorsal aorta and mostly absent from the posterior and anterior cardinal veins except for the caudal vein plexus region which showed GFP fluorescence (Fig. 1 C,E). Similar endothelial-specific expression pattern was also observed during later stages at least until $5 \mathrm{dpf}$ (data not shown). No expression was observed within the caudal fin vasculature of adult Et(GBT-B1)tpl1 zebrafish (data not shown). Many embryos showed mosaic GFP expression pattern where only some of the endothelial cells displayed GFP fluorescence. The observed mosaicism is likely caused by expression silencing due to DNA methylation which has been previously observed in transgenic lines that contain synthetic 14x Gal4-UAS sequences (Goll et al., 2009). Therefore only the embryos that showed the most robust GFP expression were propagated.

As determined by inverse PCR, the transgenic reporter was inserted within the chromosome 2, $23.7 \mathrm{~kb}$ upstream of aqp1a gene (Fig. 1F, Suppl. Fig. S2). Therefore we hypothesized that endothelial-specific enhancer of aqp1a was present in the vicinity of GFP insertion and thus resulted in endothelial GFP expression pattern. To confirm this, we investigated embryonic expression pattern of aqp1a, the sequence and protein structure of which have been previously described (Tingaud-Sequeira etal., 2008). As determined by in situ hybridization, aqp 1a is expressed in vascular endothelial cell progenitors as early as the 18-22-somite stages (Fig. 2A, and data not shown). At the 22-somite - 26 hpf stages, aqp1a expression is restricted to vascular endothelial cells within the dorsal aorta (DA), intersegmental vessels and some of the cranial vasculature including the lateral dorsal aorta (LDA), the primitive internal carotid artery (PICA), the primordial midbrain channel (PMBC), the middle cerebral vein (MCeV) and the cranial division of the internal carotid artery (CrDI) (Fig. $2 \mathrm{~A}-\mathrm{D}$ ). In addition, 
aqp1a is expressed in the hematopoietic cells within the posterior intermediate cell mass (ICM) region which mostly include erythroid progenitors (Fig. 2 A-D). By 26 hpf aqp1a expression is present in only few erythroid cells in the ICM region, because most erythroid progenitors have entered circulation at this stage (Fig. 2D). Interestingly, no aqp1a expression was observed within the posterior cardinal vein (PCV) or the primordial hindbrain channel (PHBC) while some cranial venous vessels including the $\mathrm{MCeV}$ and the $\mathrm{PMBC}$ show aqp1a expression. At 30-48 hpf stages, aqp1a is strongly expressed in the newly forming vessels such as the dorsal longitudinal anastomotic vessel (DLAV) while its expression within the DA is less intense, particularly by $48 \mathrm{hpf}$ (Fig. $2 \mathrm{E}-\mathrm{G})$. At $48 \mathrm{hpf}$ aqp1a expression is also apparent in intersegmental and cranial vessels and excluded from the PCV, similar to the earlier stages (Fig. 2 F,G). By $74 \mathrm{hpf}$ aqp1a expression is mostly restricted to endothelial cells within the secondary vessels including the DLAV, the ISVs and the aortic arches (aa) (Fig. 2H). In addition, aqp1a is expressed in a subset of neural cells within the retina and the midbrain-hindbrain region, the dorsal part of the otic vesicle and ionophores (Fig. $2 \mathrm{H}, \mathrm{I}$ ). Aqp 1a expression is also apparent within the swim bladder and the pneumatic duct (Fig. 2H, inset). By 4 $\mathrm{dpf}$ aqp1a expression is apparent in the endothelial cells of ISVs and the definitive hematopoietic progenitors within the posterior blood island region (Fig. 2J). In addition, aqp1a is expressed in the swim bladder, the intestine and neural cells in the midbrain and hindbrain regions (Fig. $2 \mathrm{~J}, \mathrm{~K}$ ).

To confirm that aqp1a is expressed in both endothelial and hematopoietic cells, we analyzed its expression in gata1 and etsrp morpholino (MO)-injected embryos (morphants). Gata1 knockdown results in the inhibition of erythropoiesis and loss of expression of multiple erythroid-specific genes (Galloway et al., 2005, Rhodes et al., 2005). Erythroid-specific aqp1a expression was absent in gata 1 morphants at $23 \mathrm{hpf}$ while its endothelial expression was not affected (Fig. 2L). It is apparent that endothelial aqp1 a expression in gata1 morphants is restricted to the DA and absent from the PCV (Fig. 2L). Based on the previous studies, expression of multiple endothelial-specific genes is downregulated in the absence of etsrp function (Pham et al., 2007, Sumanas and Lin, 2006). Endothelial specific aqp 1a expression was absent in etsrp morphants at $23 \mathrm{hpf}$ while erythroid expression was not affected (Fig. 2M). These data argue that endothelial and hematopoietic expression domains of aqp1a are controlled by different signaling pathways and require etsrp and gata1 function, respectively.

\section{Discussion}

Based on our findings, vascular endothelial aqp1a expression is restricted to the DA and excluded from the PCV. In addition, we show that aqp 1a is expressed in erythroid progenitors as early as $24 \mathrm{hpf}$ stage. While this manuscript was in preparation, an independent study was published describing zebrafish aqp1a expression pattern (Chen et al., 2010). In the latter study, it is reported that aqp1ais expressed in both DA and PCV while hematopoietic aqp1a expression was not observed until $48 \mathrm{hpf}$ stage. Because erythroid
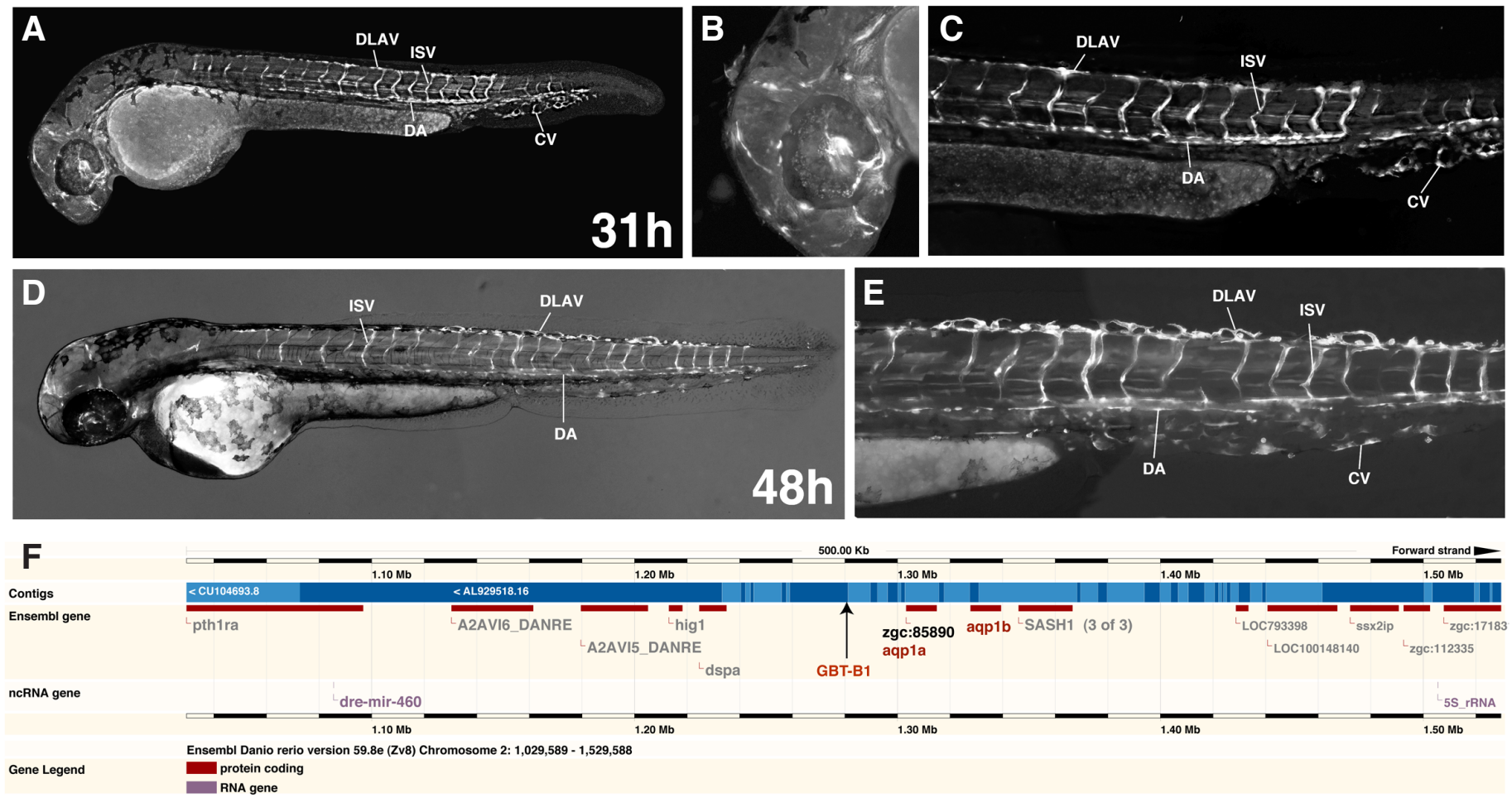

Fig. 1. Et(GBT-B1)tpl1 enhancer trap line shows endothelial-specific GFP expression pattern. (A-E) Et(GBT-B1)tp/1 line displays GFP expression in the dorsal aorta (DA), intersegmental vessels (ISV), the dorsal longitudinal anastomotic vessel (DLAV), the plexus region of the caudal vein (CV) and the cranial vasculature at $31 \mathrm{~h}(\mathbf{A}-\mathbf{C})$ and $48 \mathrm{hpf}(\mathbf{D}, \mathbf{E})$ stages. $5 \times(\mathbf{A}, \mathbf{D})$ and 10x lateral views of the head $(\mathbf{B})$, trunk and tail regions $(\mathbf{C}, \mathbf{E})$ are shown. Note that GFP fluorescence is absent from the posterior cardinal vein. GFP expression displays some mosaicism, particularly in the cranial vessels. (F) Map Viewer diagram shows that GBT-B1 insertion is located $23.7 \mathrm{~kb}$ upstream of aqp1a gene within the chromosome 2 based on Zv8 zebrafish genome assembly. The schematic location of aqp1b was added manually since it was not shown in Zv8 annotation. 
and PCV staining can overlap prior to the onset of circulation, it may be difficult to distinguish the two cell types based on solely microscopic observations. Our results obtained using gata1 MO clearly show that aqp1a is expressed in erythroid progenitors but not PCV during early embryogenesis.

Interestingly, Et(GBT-B1)tpl1 expression closely recapitulates endothelial expression pattern of the endogenous aqp 1atranscript yet it is not observed in hematopoietic cells. It is likely that endothelial and hematopoietic expression domains of aqp1a are controlled by different enhancers, consistent with gata1 and etsrpMO knockdown results. The transgenic insertion is likely located in the vicinity of the endothelial but not hematopoietic aqp1a enhancer resulting in the observed endothelial GFP expression pattern.

While zebrafish endothelial aqp1a expression is present in the dorsal aorta at $24 \mathrm{hpf}$, it becomes restricted mostly to microvasculature including intersegmental vessels by $2 \mathrm{dpf}$. Such expression pattern is also observed in adult mice and rats where AQP1 expression is restricted to microvascular endothelia (Hasegawa et al., 1994, Nielsen et al., 1993a). It is intriguing that zebrafish aqp1a expression is also observed in both primitive and definitive hematopoietic cells. AQP1 is one of the major membrane components in human erythrocytes where it is thought to transport $\mathrm{CO} 2$ across the membrane (Prasad etal., 1998, Preston and Agre, 1991). Erythroid-specific expression pattern of zebrafish aqp1a suggests that its transport function is likely to be evolutionarily conserved. Aqp1 expression has also been reported in the adult intestinal epithelia of multiple fish species (Aoki et al., 2003, Martinez et al., 2005, Raldua et al., 2008). Similar to the other species, zebrafish
Fig. 2. Aquaporin 1a is expressed in vascular endothelial and erythroid progenitors at the 22-somite - $\mathbf{9 6} \mathbf{~ h p f ~ s t a g e s . ~ ( A - G ) ~ A t ~ t h e ~}$ 22-somite-48 hpf stages aqp 1a is expressed in vascular endothelial cells that include the dorsal aorta (DA), intersegmental vessels (ISV), the dorsal longitudinal anastomotic vessel (DLAV), the lateral dorsal aorta (LDA), the primitive internal carotid artery (PICA), the primordial midbrain channel (PMBC), the primordial hindbrain channel (PHBC) and the cranial division of the internal carotid artery (CrDI). In addition, aqp1a expression is observed in hematopoietic cells $(\mathrm{HC})$ in the posterior intermediate cell mass region that mostly include erythroid progenitors. Note that aqp1a expression is absent from the trunk venous vasculature including the posterior cardinal vein. $\mathbf{( H , I ) ~ A t ~} 74$ hpfaqp1a is expressed in the endothelial cells of the secondary vessels including the DLAV, the ISV as well as the aortic arches (aa). In addition, aqp1a is expressed within the swim bladder (sb), the pneumatic duct (pd), ionocytes (io), dorsal part of the otic vesicle (ear), a subset of retinal cells (ret) and a group of cells in the midbrain and the anterior hindbrain regions (arrowhead). (H) $5 x$ lateral view; (I) 10x lateral view of the head region of the same embryo. Inset, $(H), 10 x$ ventral view of the mid-trunk region, anterior is to the left; embryo has been flat-mounted with the yolk removed. (J,K) At 96 hpf aqp1a is expressed in the endothelial cells of ISVs and definitive hematopoietic clusters $(H C$, inset, 10x view of the ventral tail region) in the posterior blood island. In addition, the expression is apparent in the swim bladder (sb), the intestine (in) and neural cells in the midbrain-hindbrain region (arrowhead). (K) Magnified lateral view of the trunk region, anterior is to the left. (L) Aqp1a erythroid expression is lost in gata1 morphants while endothelial expression is not affected. Note that aqp1a expression in the trunk region (magnified view, inset) is restricted to the DA and absent from the PCV. (M) Endothelial aqp1a expression is absent in etsrp morphant embryos at 23 hpf (inset, magnified view of the trunk region) while hematopoietic expression is not affected.
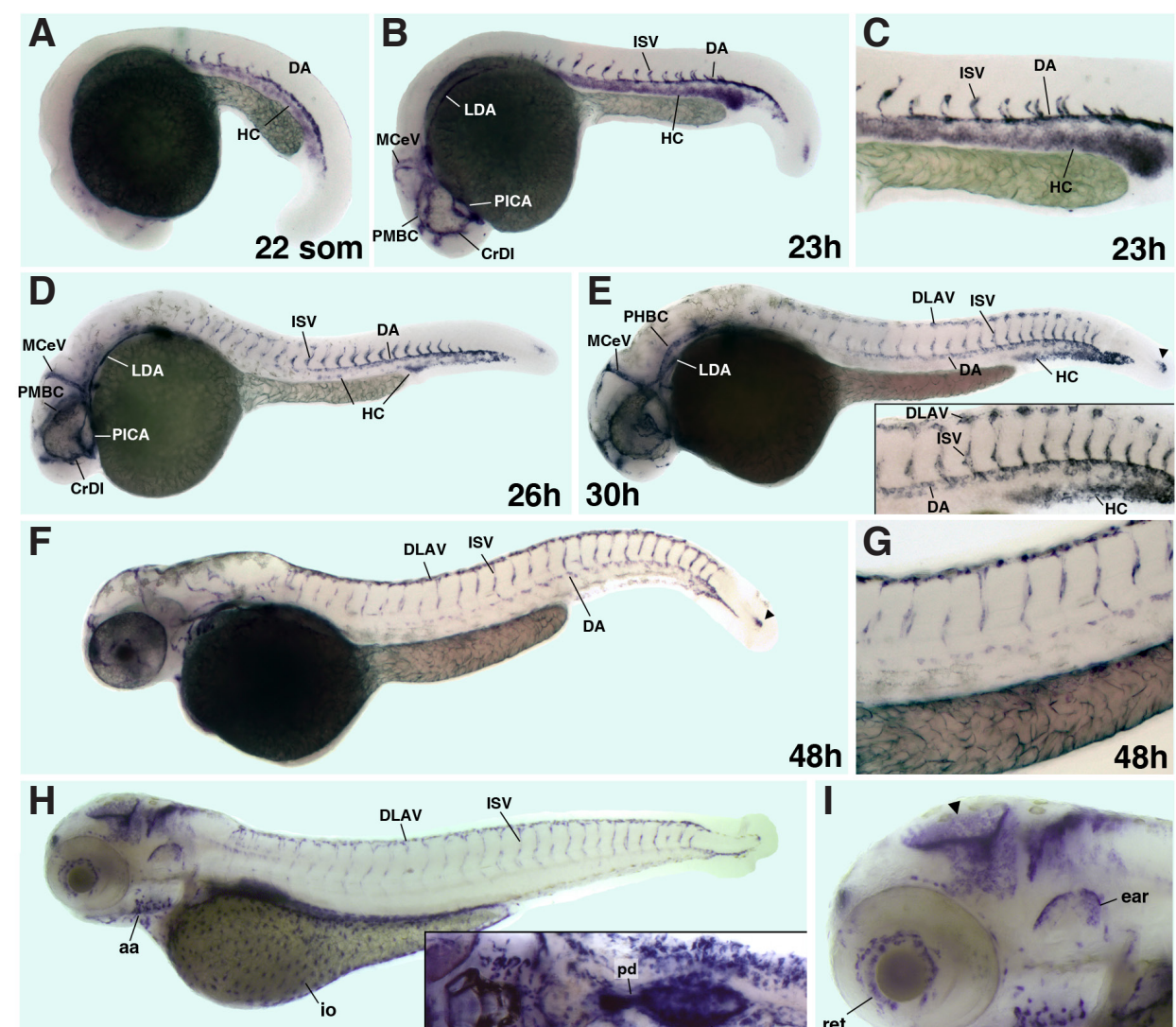

\section{$74 h$}

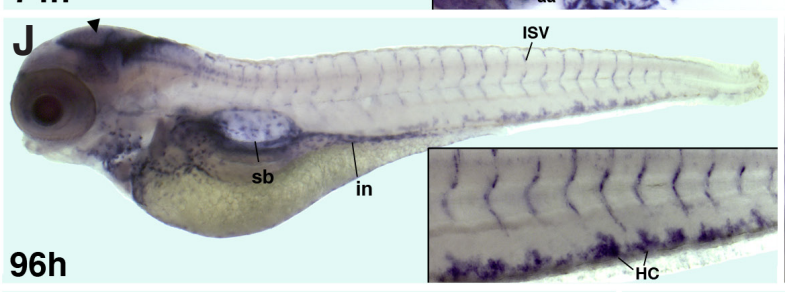

Isv
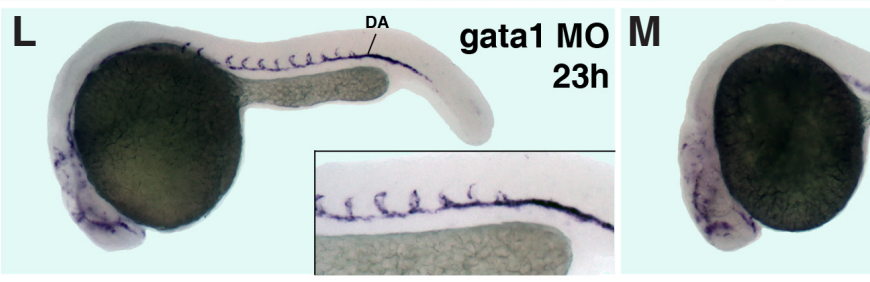

48
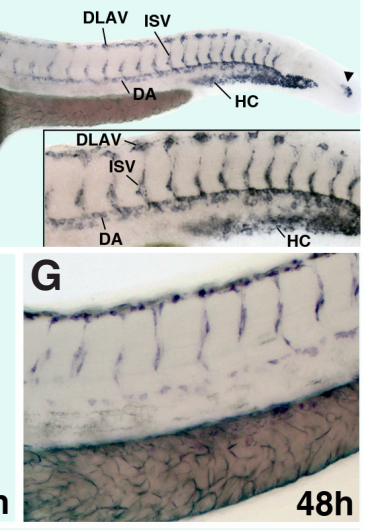

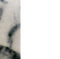

(

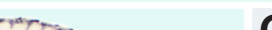


aqp1a expression is apparent in the developing intestine by $4 \mathrm{dpf}$. In summary, our study describes generation of a novel transgenic line which expresses reporter GFP under endothelial-specific aqp1a enhancer in the arterial but not venous axial vasculature. We further characterize aqp1a expression pattern which includes erythroid progenitors and vascular endothelial cells of the dorsal aorta as well as the secondary and cranial vasculature. Generation of Et(GBT-B1)tpl1 transgenic line and characterization of aqp1a expression pattern will be useful for future studies of endothelial, hematopoietic and arterial-venous differentiation. The observed aqp1aexpression pattern raises questions about possible functional roles for aqp1a during early endothelial or hematopoietic development that may be answered in the future studies.

\section{Materials and Methods}

\section{Generation of Et(GBT-B1)tpl1 line}

To generate transgenic embryos, tol2 transposase mRNA was microinjected together with GBT-B1 vector which is a dual purpose eGFP enhancer trap and Gal4-VP16 gene trap vector (Suppl. Fig. S1). The key components of the vector are a gene trap cassette based on GBT-R15 vector (Petzold et al., 2009) with AUG-less mRFP replaced by AUG-less Gal4-VP16 (Koster and Fraser, 2001). In addition, the vector has a 14XUAS:eGFP (Koster and Fraser, 2001) flanked by FRT sites. The gene enhancer trap cassette is flanked by miniTol2 transposon sites (Balciunas et al., 2006).

\section{Inverse PCR}

To identify the integration site, Et(GBT-B1)tpl1 embryos were sorted for GFP expression at 1-3 dpf. Batches of 20 GFP-positive and GFP-negative embryos were frozen at $5 \mathrm{dpf}$. Both sets of embryos were used for inverse PCR using our published protocols (Balciunas et al., 2006, Hermanson et al., 2004, Petzold et al., 2009) with Tol2-R4 (ATAATACTTAAGTACAGTAATCAAG) ant Tol2-F13 (GTACTTATTTTTTGGAGATCACTTC) primers for the first reaction and Tol2-R5 (TAATCAAGTAAAATTACTCAAGTAC) and Tol2-F11 (CCCTTGCTATTACCAAACCAATTGA) for the nested PCR step, and inverse PCR products were separated on agarose gel. PCR fragments were subsequently purified using GENJet Gel extraction kit (Fermentas) and sequenced with Tol2-R5 primer. Integration we confirmed by PCR using primers Tol2-R3 (ACTGGGCATCAGCGCAATTCAATTG) and 3B1-F1 (CTCGGAGTGCATCGAGTTCAGAA), and resulting PCR fragment was sequenced with Tol2-R3. The genomic sequence next to the integration site is shown in Suppl. Fig. S2.

\section{In situ hybridization}

cDNA clone corresponding to aqp1a gene was obtained from Open Biosystems (cat. No. EDR1052-7534520). The cDNA sequence contained full aqp1a coding sequence including 5' and 3'-UTRs and matched the aqp1areference sequence NM_207059.1. To synthesize DIG-labeled probe for in situ hybridization, pExpress-1 vector containing aqp1a clone was linearized with Smal (Fermentas) and transcribed with T7 RNA polymerase (Promega) in the presence of DIG-UTP (Roche). In situ hybridization was performed as previously described (Jowett, 1999).

\section{Imaging}

Live Et(GBT-B1)tpl1 embryos were dechorionated and mounted in $2 \%$ methylcellulose in the presence of $0.002 \%$ Tricaine (Sigma). Embryos were imaged under $5 x$ and $10 x$ objectives (Plan-Neofluar $5 x / 0.16$ NA and $10 x / 0.3$ NA) with an epifluorescent microscope (Axiolmager, Zeiss). Monochrome camera (AxioCam MRm, Zeiss) and AxioVision 4.6 software (Zeiss) was used to capture Z-stacks of fluorescent images. Projection images were obtained using Extended Focus software module (Zeiss).

To capture images after in situ hybridization, embryos were mounted in $2 \%$ methylcellulose and imaged under $10 x$ objective (Plan-Neofluar 10x/0.3, Zeiss) and an Axiolmager compound microscope using color
Axiocam Icc3 camera (Zeiss). Multiple focal planes were collected and the projection image was produced using Extended Focus module within AxioVision 4.6 software.

\section{Morpholino knockdown}

To knockdown etsrp function, $10 \mathrm{ng}$ total of 1:1 etsrp MO1 and etsrp MO2 mixture (Sumanas and Lin, 2006) was injected at the 1-2 cell stages. For gata1 knockdown, 8 ng of translation-blocking gata1 MO (Open Biosystems, sequence TTCTCCATCTGCAAGTGTAGTATTG) was used.

\section{Acknowledgements}

We thank Stephen C. Ekker for support during GBT-B1 vector construct design and initial testing. This work was supported by Cincinnati Children's Research Foundation Trustee Award to S. Sumanas.

\section{References}

AOKI, M., KANEKO, T., KATOH, F., HASEGAWA, S., TSUTSUI, N. and AIDA, K (2003). Intestinal water absorption through aquaporin 1 expressed in the apical membrane of mucosal epithelial cells in seawater-adapted Japanese eel. $J$ Exp Biol 206: 3495-3505.

ASAKAWA, K., SUSTER, M.L., MIZUSAWA, K., NAGAYOSHI, S., KOTANI, T., URASAKI, A., KISHIMOTO, Y., HIBI, M. and KAWAKAMI, K. (2008). Genetic dissection of neural circuits by Tol2 transposon-mediated Gal4 gene and enhancer trapping in zebrafish. Proc Natl Acad Sci USA 105: 1255-1260.

BALCIUNAS, D., DAVIDSON, A.E., SIVASUBBU, S., HERMANSON, S.B., WELLE, Z. and EKKER, S.C. (2004). Enhancer trapping in zebrafish using the Sleeping Beauty transposon. BMC Genomics 5: 62

BALCIUNAS, D., WANGENSTEEN, K.J., WILBER, A., BELL, J., GEURTS, A., SIVASUBBU, S., WANG, X., HACKETT, P.B., LARGAESPADA, D.A., MCIVOR, R.S. et al., (2006). Harnessing a high cargo-capacity transposon for genetic applications in vertebrates. PLoS Genet 2: e169.

BONDY, C., CHIN, E., SMITH, B.L., PRESTON, G.M. and AGRE, P. (1993). Developmental gene expression and tissue distribution of the CHIP28 water-channel protein. Proc Natl Acad Sci USA 90: 4500-4504.

CAMERINO, G.M., NICCHIA, G.P., DINARDO, M.M., RIBATTI, D., SVELTO, M. and FRIGERI, A. (2006). In vivo silencing of aquaporin-1 by RNA interference inhibits angiogenesis in the chick embryo chorioallantoic membrane assay. Cell Mol Biol (Noisy-le-grand) 52: 51-56.

CHEN, L.M., ZHAO, J., MUSA-AZIZ, R., PELLETIER, M.F., DRUMMOND, I.A. and BORON, W.F. (2010). Cloning and characterization of a zebrafish homologue of human AQP1: A bifunctional water and gas channel. Am J Physiol Regul Integr Comp Physiol. 295:R1163-1174.

ELLINGSEN, S., LAPLANTE, M.A., KONIG, M., KIKUTA, H., FURMANEK, T., HOIVIK, E.A. and BECKER, T.S. (2005). Large-scale enhancer detection in the zebrafish genome. Development 132: 3799-3811.

ENDO, M., JAIN, R.K., WITWER, B. and BROWN, D. (1999). Water channel (aquaporin 1) expression and distribution in mammary carcinomas and glioblastomas. Microvasc Res 58: 89-98.

GALLOWAY, J.L., WINGERT, R.A., THISSE, C., THISSE, B. and ZON, L.I. (2005) Loss of gata1 but not gata2 converts erythropoiesis to myelopoiesis in zebrafish embryos. Dev Cell 8: 109-116.

GOLL, M.G., ANDERSON, R., STAINIER, D.Y., SPRADLING, A.C. and HALPERN M.E. (2009). Transcriptional silencing and reactivation in transgenic zebrafish Genetics 182: 747-755.

HARRIS, H.W., JR., STRANGE, K. and ZEIDEL, M.L. (1991). Current understanding of the cellular biology and molecular structure of the antidiuretic hormone-stimulated water transport pathway. J Clin Invest 88: 1-8.

HASEGAWA, H., LIAN, S.C., FINKBEINER, W.E. and VERKMAN, A.S. (1994) Extrarenal tissue distribution of CHIP28 water channels by in situ hybridization and antibody staining. Am J Physiol 266: C893-903.

HERMANSON, S., DAVIDSON, A.E., SIVASUBBU, S., BALCIUNAS, D. and EKKER, S.C. (2004). Sleeping Beauty transposon for efficient gene delivery. Methods Cell Biol 77: 349-362.

HONG, C.C., PETERSON, Q.P., HONG, J.Y. and PETERSON, R.T. (2006). Artery/ vein specification is governed by opposing phosphatidylinositol-3 kinase and MAP 


\section{K. Rehn et al.}

kinase/ERK signaling. Curr Biol 16: 1366-1372.

JIN, S.W., BEIS, D., MITCHELL, T., CHEN, J.N. and STAINIER, D.Y. (2005). Cellular and molecular analyses of vascular tube and lumen formation in zebrafish. Development 132: 5199-5209.

JOWETT, T. (1999). Analysis of protein and gene expression. Methods Cell Biol 59: $63-85$.

KOSTER, R.W. and FRASER, S.E. (2001). Tracing transgene expression in living zebrafish embryos. Dev Biol 233: 329-346.

LAWSON, N.D., SCHEER, N., PHAM, V.N., KIM, C.H., CHITNIS, A.B., CAMPOSORTEGA, J.A. and WEINSTEIN, B.M. (2001). Notch signaling is required for arterial-venous differentiation during embryonic vascular development. Development 128: 3675-3683.

LAWSON, N.D., VOGEL, A.M. and WEINSTEIN, B.M. (2002). sonic hedgehog and vascular endothelial growth factor act upstream of the Notch pathway during arterial endothelial differentiation. Dev Cell 3: 127-136.

MARTINEZ, A.S., CUTLER, C.P., WILSON, G.D., PHILLIPS, C., HAZON, N. and CRAMB, G. (2005). Regulation of expression of two aquaporin homologs in the intestine of the European eel: effects of seawater acclimation and cortisol treatment. Am J Physiol Regul Integr Comp Physiol 288: R1733-1743.

NIELSEN, S., SMITH, B.L., CHRISTENSEN, E.I. and AGRE, P. (1993a). Distribution of the aquaporin $\mathrm{CHIP}$ in secretory and resorptive epithelia and capillary endothelia. Proc Natl Acad Sci USA 90: 7275-7279.

NIELSEN, S., SMITH, B.L., CHRISTENSEN, E.I., KNEPPER, M.A. and AGRE, P. (1993b). CHIP28 water channels are localized in constitutively water-permeable segments of the nephron. J Cell Biol 120: 371-383.

PARINOV, S., KONDRICHIN, I., KORZH, V. and EMELYANOV, A. (2004). Tol2 transposon-mediated enhancer trap to identify developmentally regulated zebrafish genes in vivo. Dev Dyn 231: 449-459.

PETZOLD, A.M., BALCIUNAS, D., SIVASUBBU, S., CLARK, K.J., BEDELL, V.M., WESTCOT, S.E., MYERS, S.R., MOULDER, G.L., THOMAS, M.J. and EKKER, S.C. (2009). Nicotine response genetics in the zebrafish. Proc Natl Acad Sci USA 106: $18662-18667$.

PHAM, V.N., LAWSON, N.D., MUGFORD, J.W., DYE, L., CASTRANOVA, D., LO, B. and WEINSTEIN, B.M. (2007). Combinatorial function of ETS transcription factors in the developing vasculature. Dev Biol 303: 772-783.

POON, K.L., LIEBLING, M., KONDRYCHYN, I., GARCIA-LECEA, M. and KORZH, V. (2010). Zebrafish cardiac enhancer trap lines: new tools for in vivo studies of cardiovascular development and disease. Dev Dyn 239: 914-926.

PRASAD, G.V., COURY, L.A., FINN, F. and ZEIDEL, M.L. (1998). Reconstituted aquaporin 1 water channels transport $\mathrm{CO} 2$ across membranes. J Biol Chem 273: 33123-33126

PRESTON, G.M. and AGRE, P. (1991). Isolation of the cDNA for erythrocyte integral membrane protein of 28 kilodaltons: member of an ancient channel family. Proc Natl Acad Sci USA 88: 11110-11114.

RALDUA, D., OTERO, D., FABRA, M. and CERDA, J. (2008). Differential localization and regulation of two aquaporin-1 homologs in the intestinal epithelia of the marine teleost Sparus aurata. Am J Physiol Regul Integr Comp Physiol 294: R993-1003.

RHODES, J., HAGEN, A., HSU, K., DENG, M., LIU, T.X., LOOK, A.T. and KANKI, J.P. (2005). Interplay of pu.1 and gata1 determines myelo-erythroid progenitor cell fate in zebrafish. Dev Cell 8: 97-108.

RIBATTI, D., FRIGERI, A., NICO, B., NICCHIA, G.P., DE GIORGIS, M., RONCALI, L. and SVELTO, M. (2002). Aquaporin-1 expression in the chick embryo chorioallantoic membrane. Anat Rec 268: 85-89.

SAADOUN, S., PAPADOPOULOS, M.C., DAVIES, D.C., BELL, B.A. and KRISHNA, S. (2002). Increased aquaporin 1 water channel expression in human brain tumours. Br J Cancer 87: 621-623.

SAADOUN, S., PAPADOPOULOS, M.C., HARA-CHIKUMA, M. and VERKMAN, A.S. (2005). Impairment of angiogenesis and cell migration by targeted aquaporin-1 gene disruption. Nature 434: 786-792.

SIVASUBBU, S., BALCIUNAS, D., DAVIDSON, A.E., PICKART, M.A., HERMANSON S.B., WANGENSTEEN, K.J., WOLBRINK, D.C. and EKKER, S.C. (2006). Genebreaking transposon mutagenesis reveals an essential role for histone H2afza in zebrafish larval development. Mech Dev 123: 513-529.

SUMANAS, S., JORNIAK, T. and LIN, S. (2005). Identification of novel vascular endothelial-specific genes by the microarray analysis of the zebrafish cloche mutants. Blood 106: 534-541.

SUMANAS, S. and LIN, S. (2006). Ets1-related protein is a key regulator of vasculogenesis in zebrafish. PLoS Biol 4: e10.

TINGAUD-SEQUEIRA, A., CALUSINSKA, M., FINN, R.N., CHAUVIGNE, F., LOZANO J. and CERDA, J. (2010). The zebrafish genome encodes the largest vertebrate repertoire of functional aquaporins with dual paralogy and substrate specificities similar to mammals. BMC Evol Biol 10: 38

TINGAUD-SEQUEIRA, A., CHAUVIGNE, F., FABRA, M., LOZANO, J., RALDUA, D. and CERDA, J. (2008). Structural and functional divergence of two fish aquaporin-1 water channels following teleost-specific gene duplication. BMC Evol Biol 8: 259

TINGAUD-SEQUEIRA, A., ZAPATER, C., CHAUVIGNE, F., OTERO, D. and CERDA, J. (2009). Adaptive plasticity of killifish (Fundulus heteroclitus) embryos: dehydration-stimulated development and differential aquaporin-3 expression. Am J Physiol Regul Integr Comp Physiol 296: R1041-1052.

VACCA, A., FRIGERI, A., RIBATTI, D., NICCHIA, G.P., NICO, B., RIA, R., SVELTO M. and DAMMACCO, F. (2001). Microvessel overexpression of aquaporin 1 parallels bone marrow angiogenesis in patients with active multiple myeloma. Br J Haematol 113: 415-421.

VERKMAN, A.S. (2006). Aquaporins in endothelia. Kidney Int 69: 1120-1123.

VERKMAN, A.S., HARA-CHIKUMA, M. and PAPADOPOULOS, M.C. (2008) Aquaporins--new players in cancer biology. J Mol Med 86: 523-529.

ZHONG, T.P., CHILDS, S., LEU, J.P. and FISHMAN, M.C. (2001). Gridlock signalling pathway fashions the first embryonic artery. Nature 414: 216-220.

ZHONG, T.P., ROSENBERG, M., MOHIDEEN, M.A., WEINSTEIN, B. and FISHMAN M.C. (2000). gridlock, an HLH gene required for assembly of the aorta in zebrafish. Science 287: 1820-1824. 


\section{Further Related Reading, published previously in the Int. J. Dev. Biol.}

Tryptase and chymase are angiogenic in vivo in the chorioallantoic membrane assay Domenico Ribatti, Girolamo Ranieri, Beatrice Nico, Vincenzo Benagiano and Enrico Crivellato Int. J. Dev. Biol. (2011) 55: 99-102

Current concepts of blood-brain barrier development Stefan Liebner, Cathrin J. Czupalla and Hartwig Wolburg Int. J. Dev. Biol. (2011) 55: 467-476

Paracrine regulation of angiogenesis by different cell types in the aorta ring model Roberto F. Nicosia, Penelope Zorzi, Giovanni Ligresti, Ann Morishita and Alfred C. Aplin Int. J. Dev. Biol. (2011) 55: 447-453

Spatially controlled expression of the Drosophila pseudouridine synthase RluA-1 Chung-Chi Wang, Jun-Chih Lo, Cheng-Ting Chien and Min-Lang Huang Int. J. Dev. Biol. (2011) 55: 223-227

The role of blood flow and microRNAs in blood vessel development Dong Liu, Janna Krueger and Ferdinand Le Noble Int. J. Dev. Biol. (2011) 55: 419-429

The Notch pathway in the developing hematopoietic system Anna Bigas, Àlex Robert-Moreno and Lluís Espinosa Int. J. Dev. Biol. (2010) 54: 1175-1188

Primitive erythropoiesis in the mammalian embryo James Palis, Jeffrey Malik, Kathleen E. McGrath and Paul D. Kingsley Int. J. Dev. Biol. (2010) 54: 1011-1018

Definitive human and mouse hematopoiesis originates from the embryonic endothelium: a new class of HSCs based on VE-cadherin expression

Estelle Oberlin, Bouchra El Hafny, Laurence Petit-Cocault and Michèle Souyri Int. J. Dev. Biol. (2010) 54: 1165-1173

The seminal work of Werner Risau in the study of the development of the vascular system

Domenico Ribatti

Int. J. Dev. Biol. (2010) 54: 567-572

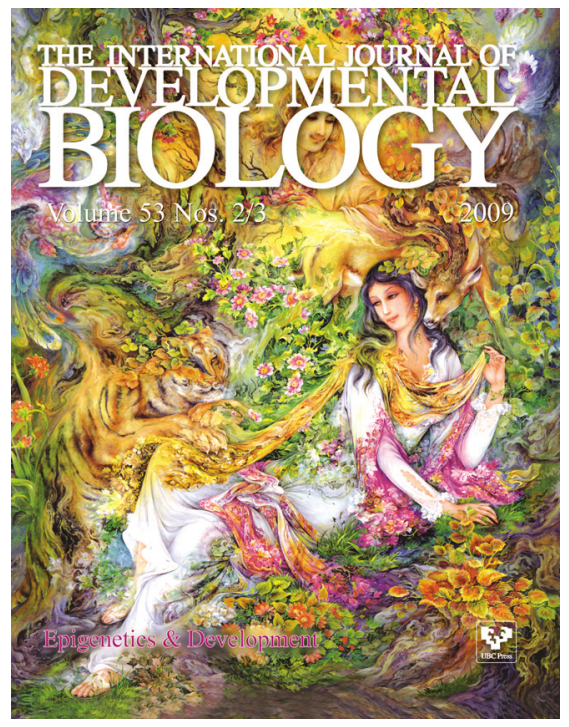

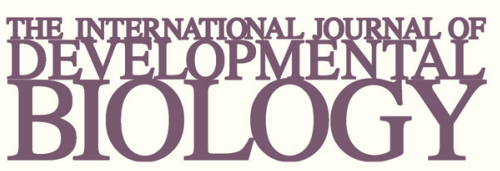

Volume 54 Nos. 6/7
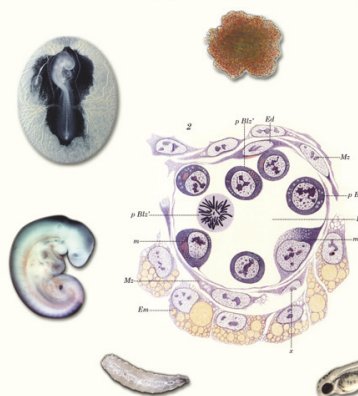

Special Issue
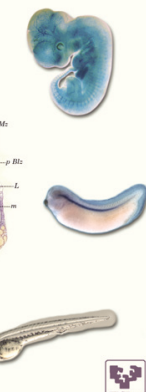

Developmental Hematopoiesis
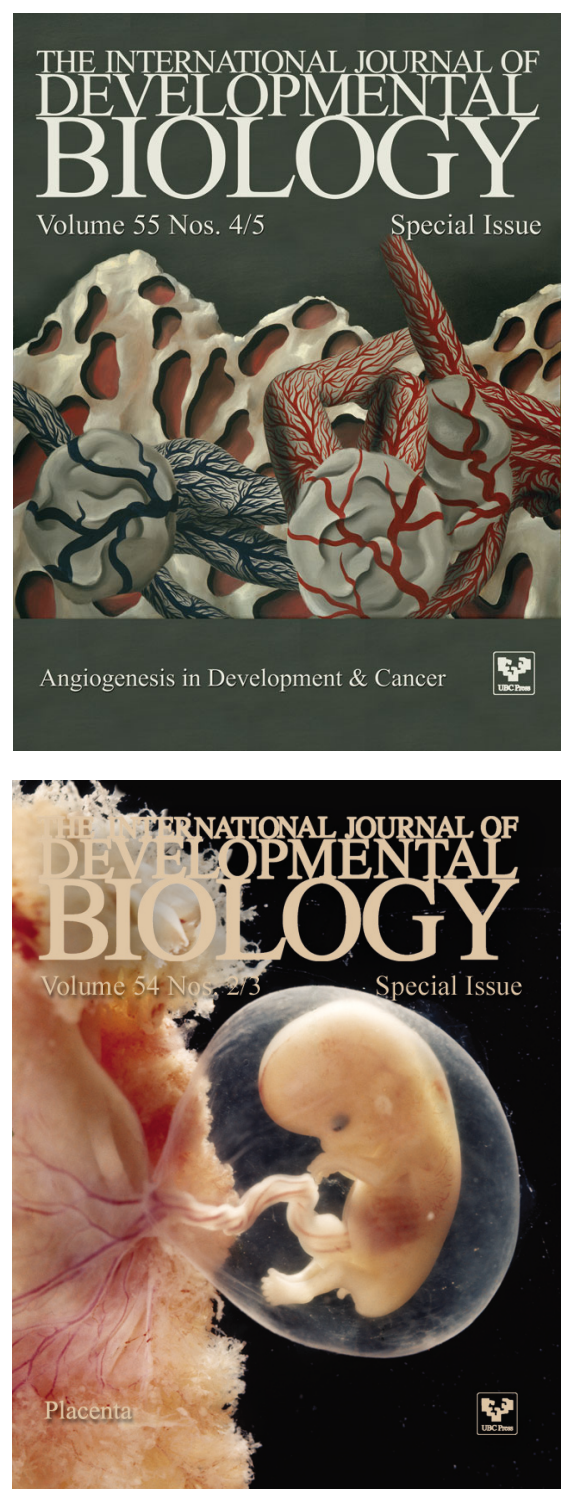